\title{
Analysis on Penetration of Ballistic Missile and Detection in Reentry Phase

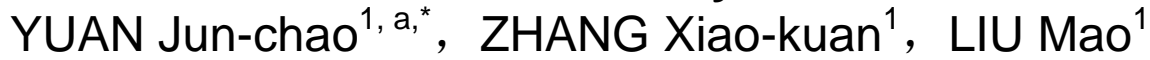 \\ ${ }^{1}$ School of Air and Missile Defense, Air Force Engineering University, Xi'an 710051, China \\ a17791252698@163.com
}

\begin{abstract}
Ballistic missile is becoming secret weapon of modern warfare because of its long distance attacks, low radar cross section, high speed and high accuracy with flexibility with nuclear warheads. In order to reduce its penetration, impact factor of ballistic missile characteristics of targets detected should be researched. Piecewise trajectory of ballistic missile is firstly described in this paper based on parts of its operational model. Then the formulas to calculate detection probability of sensor are provided and the influence of different parameters of speed, angle on changes of RCS characteristics is analyzed. Finally, the interrelation between detection probability of single sensor and reentry-angle and the interrelation between detection probability of multiple sensors and the numbers of sensors are modeled. Through the analysis, the results of the model can provide valid simulation evidence for defense of ballistic missile.
\end{abstract}

Keywords: ballistic missile, penetration, detection probability

\section{Introduction}

Ballistic missile has some advantages, such as high speed, strong mobility, small RCS, mass destruction and not easy to track. Thus, ballistic missile is important in modern war to show deterrent, and many military powers like ballistic missile. At the same time, ballistic missile brings big challenges for ground radar to defend.

This paper is organized as follows. The second chapter describes the subsection of trajectory. In the third chapter, the detection probability of the sensor to the target is studied. Then, based on the third chapter, the relationship between the RCS time series of reentry target and the detection probability of sensor is analyzed in the fourth part by simulation.

\section{Segmentation of the Trajectory [1]}

The trajectory can be divided into several sections, according to forces on ballistic missile from launching point to target point. Firstly, whether the engine and the control system work in the flight is taken as principle. According to the principle, the trajectory can be divided into two parts: phase of flight with power (active section) and phase of flight without power (passive section). This paper focuses on the passive section.

In passive section, according to the aerodynamic force on the warhead during movement, trajectory can be divided into two parts [2], section of free flight without the influence of atmosphere and reentry section which is affected by atmosphere. Because the density of air decreases with the increase of altitude, it is hard to distinguish the area with and without air. However, it is necessary to define an atmospheric boundary line or atmospheric boundary layer according to influences of the atmosphere on the flight parameters of missile. Generally speaking, ballistic missiles in medium and short range take the height of shut-down in active section as standard height for the division of free-flight phase and reentry phase, which is around $50-70 \mathrm{~km}$. For a long-range missile, the altitude around $80-100 \mathrm{~km}$ is taken as the height of atmosphere usually.

(1) Free-flight phase

The warhead in the free section is considered flying in a thin atmosphere, because the height of end point in active section is large, and the density of atmosphere decreases rapidly with the increase of height. Thus, the air force on the warhead is far less than other forces (gravity and geostrophic inertial 
force). Air power can be completely omitted. The warhead is considered to fly in the vacuum so that free-flight phase is also called vacuum section. The trajectory of free-flight section is a part of the elliptical trajectory, and the trajectory accounts for more than $80 \%-90 \%$ of the whole trajectory. The amount of free segments will be greatly increased. All the wreckage of assisted take-off rocket from the separation of the bullet, bait and missile consist of a strong target group which is hundreds of kilometers.

(2) Reentry phase

Reentry phase is the trajectory where warhead reenters the densely atmosphere. When the bullet enters the atmosphere at high speed, the speed decreases rapidly not only because warhead needs to bear strong aerodynamic heating temperature created by atmosphere and plasma layer is formed around missile, but also the warhead is under huge aerodynamic drag. Thus, the reentry trajectory has completely different characteristics from the trajectory of free-flight section.

The bullet moves freely around the center of mass at a constant angular velocity rather than keeping the posture of movement when it was separated, because the warhead is no longer affected by the aerodynamic moment and the control torque in free-flight phase. When the warhead reenters the atmosphere, free rolling-over is braking and the bullet rushes at the target stably at certain speed because the block of atmosphere increases gradually and the bullet has the aerodynamic stability and the attitude control.

During the free-flight phase which holds the longest flying time, the real warhead and decoy have the same flight characteristics, which cannot be recognized by the characteristics of trajectory [3].

\section{Probability of Detecting Targets by Sensor}

A variety of measures can be taken to penetrate when the ballistic missile reenters. Thus, great challenges are brought to the detection of ground radar. Nowadays, the capability of equipment is mainly reflected in the anti-missile operation of the ballistic missile in reentry phase. The access to information of the target mainly depends on the sensor, so the research of detection probability of sensor is particularly important.

\subsection{Target Probability of Single Sensor [4]}

The probability of single sensor to detect the target is

$$
P=1-e^{-\frac{a P_{T}}{2 z^{2} r^{4}}}
$$

In above equation, $\mathrm{P}$ is the probability of detecting target. $a$ is effective reflection area of the target. $Z$ is the RMS value of the amplitude of noise. $P_{T}$ is the power of transmitter. $r$ stands for the distance between the target and sensor.

When parameters of the sensor and characteristics of the target are determined, $\frac{a P_{T}}{2 z^{2}}$ becomes constant. If $k=\frac{a P_{T}}{2 z^{2}}$ is assumed, the probability of detecting target only has relationship with distance to the target.

$$
P=1-e^{-\frac{k}{r^{4}}}
$$

The distance is a function of time so the relationship between the probability of detecting target and the time of reentry can be found.

When there are n sensors to search space, they are assumed to be independent of each other. $P_{i}$ is detection probability of the $\mathrm{i}^{\text {th }}$ sensor. $r_{i}$ is the distance between the target and the ith sensor. $\left(x_{i}, y_{i}\right)$ is the coordinate of the ith sensor. The probability of detection by all sensors are as follows.

$$
\begin{aligned}
P_{L} & =1-\prod_{i=1}^{n}\left(1-P_{i}\right)=1-e^{-k \sum_{i=1}^{n} \frac{1}{r_{i}^{4}}} \\
& =1-e^{-k \sum_{i=1}^{n} \frac{1}{\left[\left(x-x_{i}\right)^{2}+\left(y-y_{i}\right)^{2}+z^{2}\right]^{2}}}
\end{aligned}
$$




\subsection{The Relationship between the Probability of Detection and the Number of Sensors}

In the network of sensors, the sensors can be regarded to have independent statistic. The detection of signals means that one of these sensors can detect the target. $P_{i}$ is assumed first which is the detection probability of the ith sensor. With n sensors in the network, the probability of whole network is

$$
P_{L}=1-\prod_{i=1}^{n}\left(1-P_{i}\right)
$$

The probability of different detecting sensors in the network may be the same or different. Following two conditions should be discussed which will determine the relationship between $P_{L}$ and $n$.

a. The probability of sensors in the network is the same.

$P_{1}=P_{2}=\cdots=P_{n}=P$ then

$$
P_{L}=1-\prod_{i=1}^{n}\left(1-P_{i}\right)=1-(1-P)^{n}
$$

In order to meet the requirement of total detection probability in the network, $P_{L} \geq P_{0}$. The amount of sensors, $\mathrm{n}$, should satisfy that

$$
n \geq \ln \left(1-P_{0}\right) / \ln (1-P)
$$

b. The probability of sensors in the network is different.

According to equation (4), because $P_{L} \geq P_{0}$, the total detection probability required by are sensor should satisfy that

$$
1-\prod_{i=1}^{n}\left(1-P_{i}\right) \geq P_{0}
$$

Then, the amount of sensors, $n$, should satisfy that

$$
n \geq 1+\left|\ln \prod_{i=1}^{n} \frac{1-P_{i}}{1-P_{0}} / \ln \left(1-P_{0}\right)\right|
$$

\section{Simulation Results and Analysis of Reentry Stage}

In order to study the detection probability of sensor to the target at reentry stage, the target RCS cannot be used as a constant technical index simply. The RCS series of the reentry warhead with the change of distance and time detected by radar must be used [5]. Fig. 1 shows electromagnetic calculation model of reentry warhead. Due to the symmetry, the azimuth angle is taken as $\varphi=0^{\circ}$, and pitch angle can be taken as $\varepsilon=0^{\circ} \sim 180^{\circ}$.

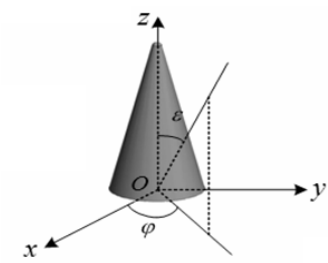

Fig. 1 Model of reentry warhead

It is assumed that the uniform plasma parameter around warhead is $\varepsilon_{r}=2.4+j 0.5$ [6], the thickness is $5 \mathrm{~cm}$, and wave frequency of the incident electromagnetic is $1 \mathrm{GHz}$. Fig. 2 shows RCS curves of warhead with and without plasma under the vertical incident wave irradiation. According to fig. 2, when the target reenters, there is plasma around target which reduces the real RCS of target obviously. At the same time, it also explains the rationality and necessity of the simulation of warhead in this paper. 


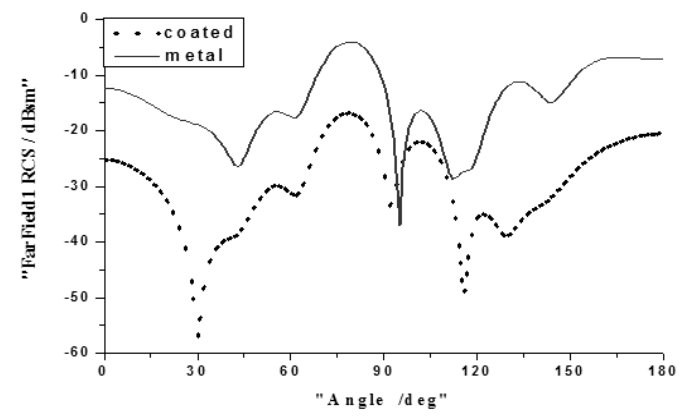

Fig. 2 RCS curves of warhead with and without plasma

Now, the relative position of warhead and ground-based radar is established. In order to simplify the problem, the route of reentry missile is assumed to be straight line. Radar, ballistic missile and attacked target are in the same vertical plane. The attitude angle of the warhead is assumed to be kept same as that of reentry. Fig. 3 shows the relative position of trajectory and radar (The effects of curvature of the earth are ignored.). In order to explain clearly, the trajectory 1 and trajectory 2 are drawn at different sides. In reality, these two trajectories are both at the side of radar station.

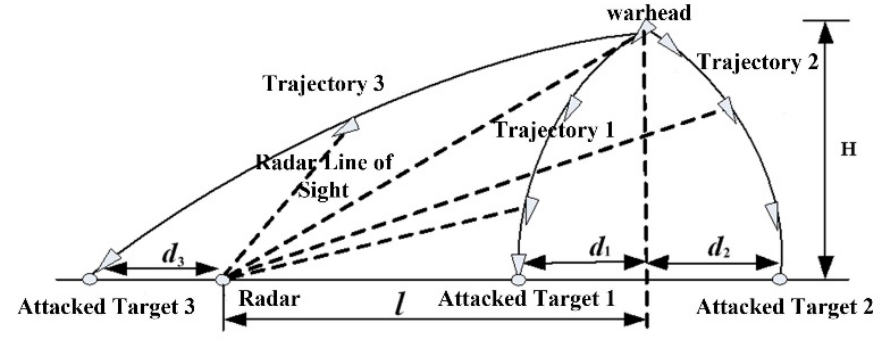

Fig. 3 Position between radar and trajectories

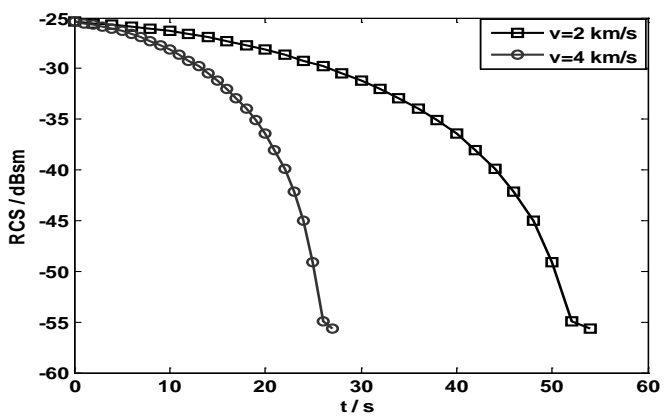

Fig. 4 RCS series at different reentry speeds

Fig. 4 shows RCS series when the angle of reentry is 30 degree, and the speeds of reentry are $2 \mathrm{~km} / \mathrm{s}$ and $4 \mathrm{~km} / \mathrm{s}$. Based on fig. 4, RCS time series have the similar tendency but the amplitude of RCS changes in different speeds with time.

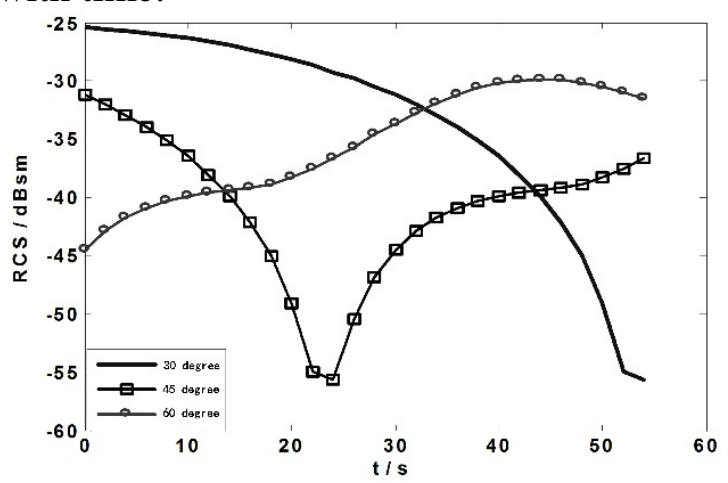

Fig. 5 RCS series at different reentry

Fig. 5 shows RCS series when the reentry speed is $2 \mathrm{~km} / \mathrm{s}$ and the angles of reentry are 30 degree, 45 degree and 60 degree respectively. According to fig. 5, with different reentry angles, RCS time series 
have obvious differences. By analyzing fig. 4 and fig. 5, dynamic RCS can provide a method to classify and recognize the target. Also, the movement characteristics of target can be distinguished. With dynamic RCS series of target, the detection probability of sensor can be calculated by the method in chapter 3 .

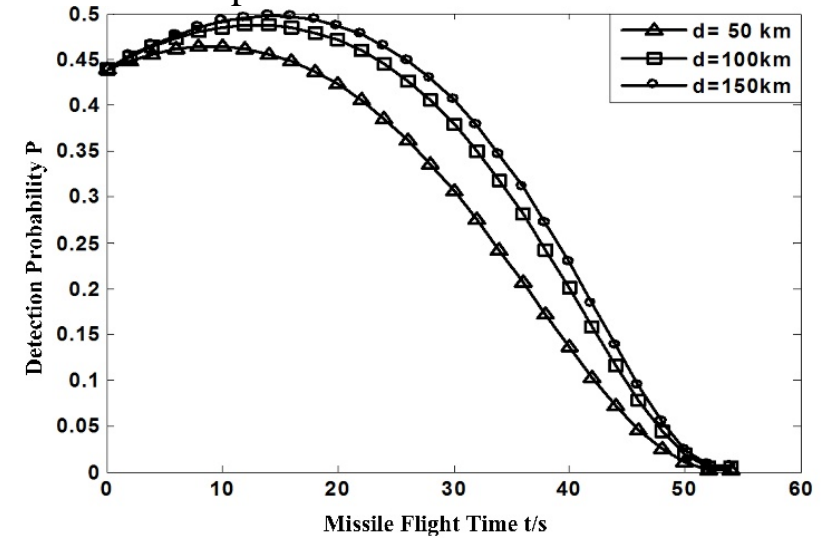

(a) reentry angle of 30 degree

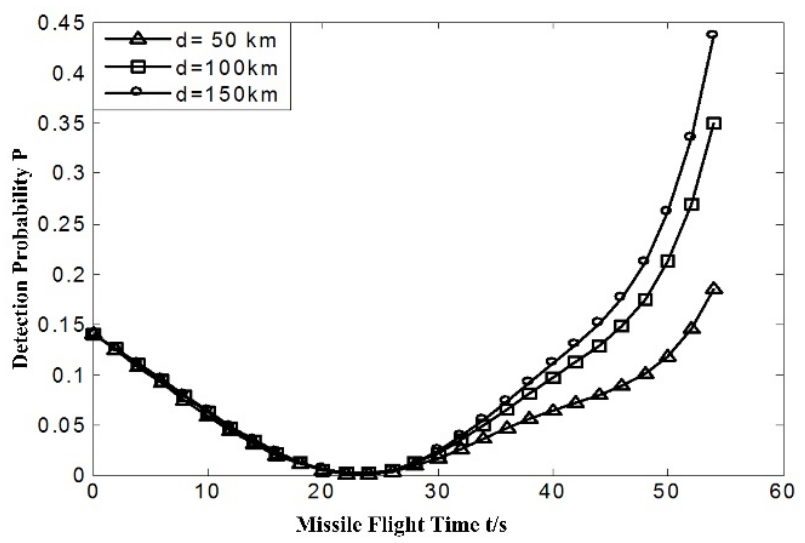

(b) reentry angle of 45 degree

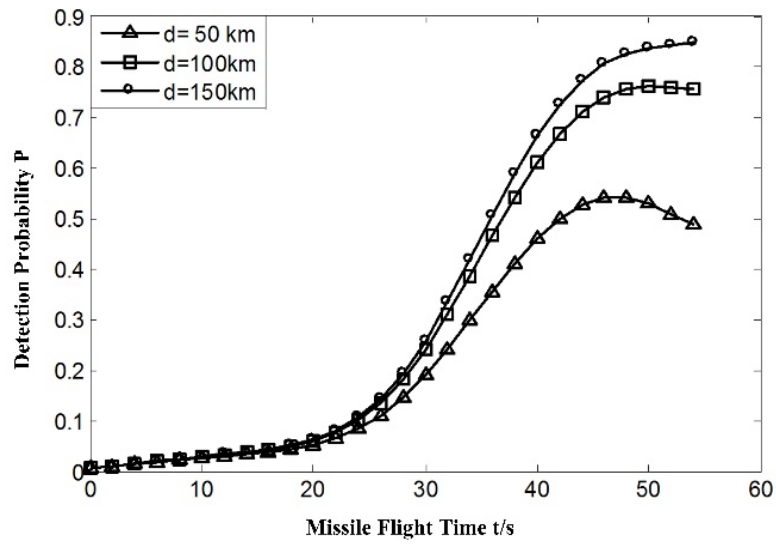

(c) reentry angle of 60 degree

Fig. 6 Tendency of detection probability P changing with distance $\mathrm{d}$

Above fig. 6 gives the relationship between detection probability P and reentry time $t$ when the speed of reentry is $2 \mathrm{~km} / \mathrm{s}$, and the reentry angles are different. When the angles of reentry are the same, the detection probability $\mathrm{P}$ has the similar tendency. When the angles of reentry are different, detection probability has obvious differences, and the best time of detection is also different. Therefore, in order to obtain the best tracking time, it is necessary to adjust the angle between the line of sight and the warhead of the missile by the method of radar maneuver.

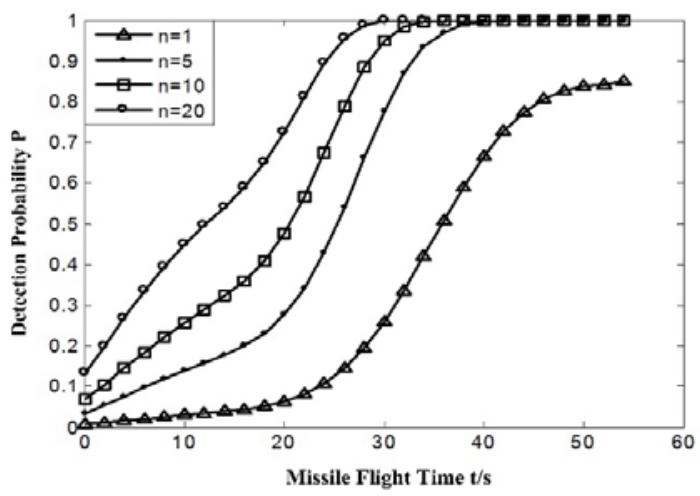

Fig. 7 Trend of detection probability $\mathrm{P}$ with the number of sensors $n$

The trend of detection probability $\mathrm{P}$ with the number of sensors $\mathrm{n}$ is shown in fig. 7 when each sensor has the same detection probability. The probability that the ballistic missile enters the reentry phase is greater when the number of sensors becomes more. However, as time goes on, the target has already 
been in the best detection area of radar. The probability of detection has nothing to do with the number of sensors so that the detection probability of ballistic missile does not increase with the increase of sensor. The number of sensors should be selected appropriately in the engineering design.

\section{Conclusions}

The detection of ballistic missile is the basis of ballistic defense. It is of great significance to understand and learn about the flight of ballistic missile in different trajectories and possible penetration measures when attacking, which leads ground air-defense weapon system to take targeted defensive measures. In this paper, based on the analysis of the ballistic trajectory, the detection probability of the sensor is calculated according to the relationship between the warhead RCS and different factors, such as reentry trajectory, reentry speed etc. The results show that this method can provide a certain basis for the ballistic missile defense.

\section{References}

[1]YUAN Ke-liang, SHI Zi-sheng, LUO Jin-liang. Analysis and Countermeasures of the Electromagnetic Threat of Ballistic Missile Penetration[J]. Shipboard Electronic Countermeasure, 2013, 36(2): 33-34.

[2] LIU Ji-zhong, WANG Xiao-dong, GAO Lei. Ballistic Missile[M]. Beijing: National Defence Industry Press, 2013.

[3] LI Shi-gang, ZHANG Li-zheng. Research on Ballistic Missile Penetration[J], Command Control \& Simulation, 2014, 6(36): 73-76.

[4] YU You-wen, CHANG Jian, CHENG Ji-hong. Principle of Sensor \& Engineering Application(Forth Edition)[M], Xian: Xian University of Electronic science and Technology Press, 2014.

[5] WANG Tong, TONG Chuang-ming, PENG Peng, et.al. Study on Radar Cross Section Time Series in Boost-phase of Ballistic Missile Based on Characteristic of Trajectory[J]. Journal of Projectiles, Rockets, Missiles and Guidance, 2014, 34(3): 106-108.

[6] ZHOU chao, ZHANG Xiao-kuan, ZHANG Chen-xin, WU Guo-cheng. A Study on the Influence on Warhead RCS of Plasma Parameters in the Reentry Phase[J]. Modern Radar, 2014, 36(3): 83-86. 